\title{
Can Social Information Affect What Job You Choose and Keep?
}

by

Lucas C. Coffman

Clayton R. Featherstone

Judd B. Kessler

\section{Online Appendix}

\section{Proof of results in Subsection 4.A:}

The fraction of admits with prior belief $\beta$ that are persuaded is

$$
\frac{1}{\sigma} \cdot \varphi\left(\frac{\beta-\mu_{\theta}}{\sigma}\right) \cdot(s-\beta) \cdot \varepsilon .
$$

Taking the expectation of this over possible prior beliefs yields the overall fraction that are persuaded, that is, the treatment effect:

$$
\begin{aligned}
T & =\mathbb{E}\left[\frac{1}{\sigma} \cdot \varphi\left(\frac{\beta-\mu_{\theta}}{\sigma}\right) \cdot(s-\beta) \cdot \varepsilon\right], \\
& =\frac{1}{\sigma^{2}} \cdot \int \varphi\left(\frac{\beta-\mu_{\beta}}{\sigma}\right) \cdot \varphi\left(\frac{\beta-\mu_{\theta}}{\sigma}\right) \cdot(s-\beta) \cdot \varepsilon \cdot d \beta .
\end{aligned}
$$

Using a well-known identity for the product of two Gaussian densities, this becomes

$$
\begin{aligned}
T & =\frac{1}{\sigma^{2}} \cdot \int \varphi\left(\frac{\mu_{\beta}-\mu_{\theta}}{\sigma \cdot \sqrt{2}}\right) \cdot \varphi\left(\frac{\beta-\frac{\mu_{\beta}+\mu_{\theta}}{2}}{\frac{\sigma}{\sqrt{2}}}\right) \cdot(s-\beta) \cdot \varepsilon \cdot d \beta, \\
& =\frac{1}{\sigma \sqrt{2}} \cdot \varphi\left(\frac{\mu_{\beta}-\mu_{\theta}}{\sigma \cdot \sqrt{2}}\right) \cdot\left(s-\frac{\mu_{\beta}+\mu_{\theta}}{2}\right) \cdot \varepsilon,
\end{aligned}
$$

which is our equation (1).

To generate equation (2), we need to have things in terms of the matriculation rate $M$. Since both $\beta$ and $\theta$ are normally distributed, we know that $M=\Phi\left(\frac{\mu_{\beta}-\mu_{\theta}}{\sigma \sqrt{2}}\right)$, which means that $\mu_{\theta}=\mu_{\beta}-\Phi^{-1}(M) \cdot \sigma \cdot \sqrt{2}$. Plugging this formula into our last expression for the treatment effect yields

$$
T(M)=\left[\frac{1}{\sqrt{2}} \cdot \varphi\left(\Phi^{-1}(M)\right)\right] \cdot\left[\left(z_{S}+\frac{\Phi^{-1}(M)}{\sqrt{2}}\right) \cdot \varepsilon\right],
$$

which is our equation (2). 


\section{Admissions}

Dear Lauren,

I am pleased to extend you an offer to join the Teach For America 2012 corps! Your acceptance into Teach For America reflects your outstanding accomplishments, leadership potential, and commitment to expanding educational opportunity for children in low-income communities. In order to secure your place in the 2012 corps, you must complete matriculation forms on the Applicant Center on or before Monday, November 21 at 6 p.m. ET

Changing children's life trajectories by effecting meaningful gains in their academic achievement is an incredibly challenging pursuit. You have demonstrated great potential to excel as a classroom leader who will work in partnership with families, schools, and communities to offer your students the educational opportunities they deserve, and we are excited to welcome you to this effort. More than 28,000 Teach For America corps members and alumni are using their unique talents, skills, and perspective to help transform education for children in low-income communities and address the factors that contribute to educational inequity, and we hope that you will join them in this important work.

On the Applicant Center, you can view a special welcome video from our Chief Executive Officer and Founder Wendy Kopp, as well as access information that will help you make an informed decision about this significant commitment, including:

- Regional and grade/subject assignment: In determining your assignment, we made the best possible match between your regional, grade-level, and subject-area preferences, the projected needs of the school districts with which we work, and the requirements necessary to teach in those districts. Since we carefully consider each applicant's qualifications and preferences when determining his or her assignment, we rarely reassign an applicant to a new region.

- Information about your region: The staff in your assigned region has posted important resources on the Applicant Center that will provide: details about living and teaching in your region; information about the summer institute; and the phone numbers and/or e-mail addresses for corps members and alumni who would be happy to answer any questions you have.

Last year, more than $84 \%$ of admitted applicants made the decision to join

the corps, and I sincerely hope you join them. If we can be of any

assistance, please contact us at admissions@teachforamerica.org.

Congratulations again, and welcome to Teach For America!

Sincerely,

Sean Waldheim

Vice President, Admissions
CHOICE

CLICKS

What will you

Teach For? Add

your voice.

Share your

acceptance on

Facebook and

see who will be

joining you in the

fight.

What role will you play? Hear an alum's story.

Join the

conversation:

选

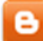

TEACHFORAMERICA

Figure A1: Admissions letter (dashed box added to highlight location of social information line) 
Table A1: Number of admitted applicants, broken down by treatment and wave

\begin{tabular}{llccccc}
\hline & & \multicolumn{3}{c}{ Wave } & \\
& & 1 & 2 & 3 & 4 & Overall \\
\hline \multirow{2}{*}{ All subjects } & Control & 790 & 840 & 791 & 916 & 3,337 \\
& Social Information & 800 & 840 & 795 & 913 & 3,348 \\
& Stratified on Disappointing Assignment? & $\mathrm{N}$ & $\mathrm{N}$ & $\mathrm{Y}$ & $\mathrm{Y}$ & \\
\hline Disappointing & Control & 197 & 216 & 319 & 427 & 1,159 \\
Assignment & Social Information & 203 & 200 & 319 & 425 & 1,147 \\
Pleasing & Control & 593 & 624 & 472 & 489 & 2,178 \\
Assignment & Social Information & 597 & 640 & 476 & 488 & 2,201 \\
\hline \multirow{2}{*}{} & Total & 1,590 & 1,680 & 1,586 & 1,829 & 6,685 \\
\hline
\end{tabular}

Balance and stratification were not perfect because the TFA data we randomized included a few "repeat" applicants who were sent multiple acceptance letters. These subjects have been dropped from all analysis. "Disappointing Assignment" refers to admits who were not offered their first choice region and first choice subject. 
Table A2: Regression of Propensity to Join and Treatment on Working for TFA over Time (including Wave-Specific treatment)

\begin{tabular}{|c|c|c|c|c|c|c|c|c|c|c|}
\hline & \multicolumn{10}{|c|}{ Linear Probability Models (in TFA at decision point $=1$ ) } \\
\hline & \multicolumn{10}{|c|}{ No subgrouping $(\mathrm{N}=8263)$} \\
\hline & \multicolumn{2}{|c|}{ Initial Commitment } & \multicolumn{2}{|c|}{ Showed to Institute } & \multicolumn{2}{|c|}{ Started Teaching } & \multicolumn{2}{|c|}{ Teaching Spring 2013} & \multicolumn{2}{|c|}{ Teaching Fall 2013} \\
\hline & $(1)$ & $(2)$ & $(3)$ & (4) & $(5)$ & $(6)$ & $(7)$ & $(8)$ & $(9)$ & $(10)$ \\
\hline Social Information & $\begin{array}{c}0.017 \\
(0.009)\end{array}$ & $\begin{array}{l}0.020 \\
(0.009)\end{array}$ & $\begin{array}{c}0.007 \\
(0.010)\end{array}$ & $\begin{array}{c}0.012 \\
(0.010)\end{array}$ & $\begin{array}{c}0.010 \\
(0.010)\end{array}$ & $\begin{array}{c}0.014 \\
(0.010)\end{array}$ & $\begin{array}{c}0.011 \\
(0.011)\end{array}$ & $\begin{array}{c}0.014 \\
(0.011)\end{array}$ & $\begin{array}{c}0.024 \\
(0.011)\end{array}$ & $\begin{array}{c}0.028 \\
(0.011)\end{array}$ \\
\hline \multirow[t]{3}{*}{ Control Mean } & 0.773 & 0.773 & 0.694 & 0.694 & 0.681 & 0.681 & 0.643 & 0.643 & 0.580 & 0.580 \\
\hline & \multicolumn{10}{|c|}{ Subgrouping by Disappointing Assignment $(\mathrm{N}=8263)$} \\
\hline & $(11)$ & $(12)$ & $(13)$ & (14) & $(15)$ & $(16)$ & $(17)$ & $(18)$ & (19) & $(20)$ \\
\hline $\begin{array}{l}\text { Social Information } \times \\
\text { Disappointing }\end{array}$ & $\begin{array}{c}0.038 \\
(0.017)\end{array}$ & $\begin{array}{c}0.042 \\
(0.017)\end{array}$ & $\begin{array}{c}0.024 \\
(0.019)\end{array}$ & $\begin{array}{c}0.029 \\
(0.018)\end{array}$ & $\begin{array}{c}0.030 \\
(0.019)\end{array}$ & $\begin{array}{c}0.035 \\
(0.019)\end{array}$ & $\begin{array}{c}0.034 \\
(0.019)\end{array}$ & $\begin{array}{c}0.038 \\
(0.019)\end{array}$ & $\begin{array}{c}0.042 \\
(0.019)\end{array}$ & $\begin{array}{c}0.045 \\
(0.019)\end{array}$ \\
\hline $\begin{array}{l}\text { Social Information } \times \\
\text { Pleasing }\end{array}$ & $\begin{array}{c}0.006 \\
(0.011)\end{array}$ & $\begin{array}{c}0.009 \\
(0.010)\end{array}$ & $\begin{array}{l}-0.001 \\
(0.012)\end{array}$ & $\begin{array}{c}0.003 \\
(0.012)\end{array}$ & $\begin{array}{l}-0.000 \\
(0.012)\end{array}$ & $\begin{array}{c}0.004 \\
(0.012)\end{array}$ & $\begin{array}{l}-0.001 \\
(0.013)\end{array}$ & $\begin{array}{c}0.003 \\
(0.013)\end{array}$ & $\begin{array}{c}0.014 \\
(0.013)\end{array}$ & $\begin{array}{c}0.019 \\
(0.013)\end{array}$ \\
\hline $\begin{array}{l}\text { Disappointing } \\
\text { Assignment }\end{array}$ & $\begin{array}{l}-0.078 \\
(0.016)\end{array}$ & $\begin{array}{l}-0.080 \\
(0.015)\end{array}$ & $\begin{array}{l}-0.090 \\
(0.017)\end{array}$ & $\begin{array}{l}-0.091 \\
(0.017)\end{array}$ & $\begin{array}{l}-0.092 \\
(0.017)\end{array}$ & $\begin{array}{l}-0.092 \\
(0.017)\end{array}$ & $\begin{array}{l}-0.091 \\
(0.018)\end{array}$ & $\begin{array}{l}-0.091 \\
(0.018)\end{array}$ & $\begin{array}{l}-0.082 \\
(0.018)\end{array}$ & $\begin{array}{l}-0.081 \\
(0.018)\end{array}$ \\
\hline \multirow[t]{3}{*}{ Pleasing Control Mean } & 0.808 & 0.808 & 0.731 & 0.731 & 0.719 & 0.719 & 0.681 & 0.681 & 0.614 & 0.614 \\
\hline & \multicolumn{10}{|c|}{ Subgrouping by Moderately Aligned $(\mathrm{N}=8263)$} \\
\hline & $(21)$ & $(22)$ & $(23)$ & $(24)$ & $(25)$ & $(26)$ & $(27)$ & $(28)$ & $(29)$ & $(30)$ \\
\hline $\begin{array}{l}\text { Social Information } \times \\
\text { Moderately Aligned }\end{array}$ & $\begin{array}{c}0.036 \\
(0.016)\end{array}$ & $\begin{array}{c}0.035 \\
(0.015)\end{array}$ & $\begin{array}{c}0.030 \\
(0.017)\end{array}$ & $\begin{array}{c}0.030 \\
(0.017)\end{array}$ & $\begin{array}{c}0.040 \\
(0.017)\end{array}$ & $\begin{array}{c}0.041 \\
(0.017)\end{array}$ & $\begin{array}{c}0.036 \\
(0.018)\end{array}$ & $\begin{array}{c}0.036 \\
(0.018)\end{array}$ & $\begin{array}{c}0.044 \\
(0.018)\end{array}$ & $\begin{array}{c}0.045 \\
(0.018)\end{array}$ \\
\hline $\begin{array}{l}\text { Social Information } \times \\
\text { Highly Aligned }\end{array}$ & $\begin{array}{c}0.004 \\
(0.011)\end{array}$ & $\begin{array}{c}0.011 \\
(0.011)\end{array}$ & $\begin{array}{l}-0.007 \\
(0.013)\end{array}$ & $\begin{array}{l}-0.000 \\
(0.013)\end{array}$ & $\begin{array}{l}-0.009 \\
(0.013)\end{array}$ & $\begin{array}{l}-0.002 \\
(0.013)\end{array}$ & $\begin{array}{l}-0.005 \\
(0.013)\end{array}$ & $\begin{array}{c}0.001 \\
(0.013)\end{array}$ & $\begin{array}{c}0.011 \\
(0.014)\end{array}$ & $\begin{array}{c}0.016 \\
(0.014)\end{array}$ \\
\hline Moderately Aligned & $\begin{array}{l}-0.073 \\
(0.015)\end{array}$ & $\begin{array}{l}-0.061 \\
(0.015)\end{array}$ & $\begin{array}{l}-0.079 \\
(0.016)\end{array}$ & $\begin{array}{l}-0.070 \\
(0.016)\end{array}$ & $\begin{array}{l}-0.087 \\
(0.017)\end{array}$ & $\begin{array}{l}-0.080 \\
(0.017)\end{array}$ & $\begin{array}{l}-0.086 \\
(0.017)\end{array}$ & $\begin{array}{l}-0.081 \\
(0.017)\end{array}$ & $\begin{array}{l}-0.077 \\
(0.017)\end{array}$ & $\begin{array}{l}-0.074 \\
(0.017)\end{array}$ \\
\hline \multirow[t]{3}{*}{$\begin{array}{l}\text { Highly Aligned } \\
\text { Control Mean }\end{array}$} & 0.803 & 0.803 & 0.726 & 0.726 & 0.717 & 0.717 & 0.678 & 0.678 & 0.611 & 0.611 \\
\hline & \multicolumn{10}{|c|}{ Subgrouping by Not Certain $(\mathrm{N}=7902)$} \\
\hline & $(31)$ & $(32)$ & (33) & $(34)$ & $(35)$ & (36) & (37) & $(38)$ & (39) & $(40)$ \\
\hline $\begin{array}{l}\text { Social Information } \times \\
\text { Not Certain }\end{array}$ & $\begin{array}{c}0.034 \\
(0.011)\end{array}$ & $\begin{array}{c}0.036 \\
(0.011)\end{array}$ & $\begin{array}{c}0.023 \\
(0.013)\end{array}$ & $\begin{array}{c}0.026 \\
(0.012)\end{array}$ & $\begin{array}{c}0.023 \\
(0.013)\end{array}$ & $\begin{array}{c}0.026 \\
(0.013)\end{array}$ & $\begin{array}{c}0.023 \\
(0.013)\end{array}$ & $\begin{array}{c}0.026 \\
(0.013)\end{array}$ & $\begin{array}{c}0.031 \\
(0.013)\end{array}$ & $\begin{array}{c}0.035 \\
(0.013)\end{array}$ \\
\hline $\begin{array}{l}\text { Social Information } \times \\
\text { Certain }\end{array}$ & $\begin{array}{l}-0.018 \\
(0.010)\end{array}$ & $\begin{array}{l}-0.016 \\
(0.010)\end{array}$ & $\begin{array}{l}-0.023 \\
(0.014)\end{array}$ & $\begin{array}{l}-0.023 \\
(0.014)\end{array}$ & $\begin{array}{l}-0.013 \\
(0.015)\end{array}$ & $\begin{array}{l}-0.012 \\
(0.015)\end{array}$ & $\begin{array}{l}-0.012 \\
(0.017)\end{array}$ & $\begin{array}{l}-0.012 \\
(0.017)\end{array}$ & $\begin{array}{c}0.017 \\
(0.019)\end{array}$ & $\begin{array}{c}0.017 \\
(0.019)\end{array}$ \\
\hline Not Certain & $\begin{array}{l}-0.190 \\
(0.012)\end{array}$ & $\begin{array}{l}-0.179 \\
(0.012)\end{array}$ & $\begin{array}{l}-0.241 \\
(0.014)\end{array}$ & $\begin{array}{l}-0.236 \\
(0.014)\end{array}$ & $\begin{array}{l}-0.228 \\
(0.015)\end{array}$ & $\begin{array}{l}-0.225 \\
(0.015)\end{array}$ & $\begin{array}{l}-0.213 \\
(0.016)\end{array}$ & $\begin{array}{l}-0.212 \\
(0.017)\end{array}$ & $\begin{array}{l}-0.184 \\
(0.018)\end{array}$ & $\begin{array}{l}-0.186 \\
(0.018)\end{array}$ \\
\hline Certain Control Mean & 0.953 & 0.953 & 0.908 & 0.908 & 0.885 & 0.885 & 0.834 & 0.834 & 0.745 & 0.745 \\
\hline Demog. Controls? & No & Yes & No & Yes & No & Yes & No & Yes & No & Yes \\
\hline
\end{tabular}

Table shows Linear Probability Model (OLS) regression results of whether the individual was working for TFA at each of the five milestones. All regressions include dummy variables for wave during which the applicant was admitted and a dummy for displeasing assignment, which was a stratifying variable. The even columns control for demographic characteristics: gender, race, ethnicity, socioeconomic status (based on whether an accepted applicant had a full, partial or no Pell grant during college), whether they were a math/science major, and their student status or profession before applying to TFA. There are fewer observations in Not Certain subgroup regressions because about $5 \%$ of admits did not respond to the survey question used to construct the subgroup. Robust standard errors are reported in parentheses. The omitted group's mean likelihood of working for TFA at that milestone is reported. 


\section{Survey questions}

All three questions measured on a scale from 1, "very unlikely", to 7, "very likely".

- Employment Prospects - "In two years' time, how much more or less likely would an employer be to hire you if that employer knew you had participated in Teach for America?"

- Graduate School Prospects - "In two years' time, how much more or less likely to be admitted to a graduate program (e.g. medical school, law school, master's degree) if that school knew you had participated in Teach for America?"

- TFA Impact on Students - "Consider two otherwise identical students, one of whom has a TFA teacher for one year, and one of whom does not. How much more or less likely is the student with the TFA teacher to succeed?" 


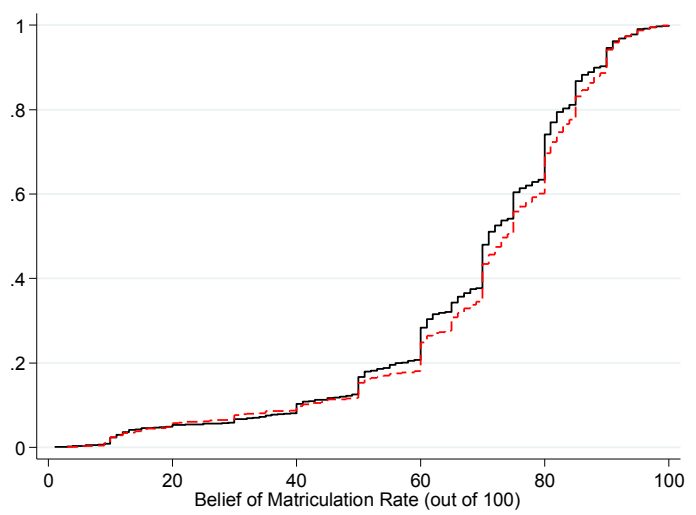

Panel A: All Admits

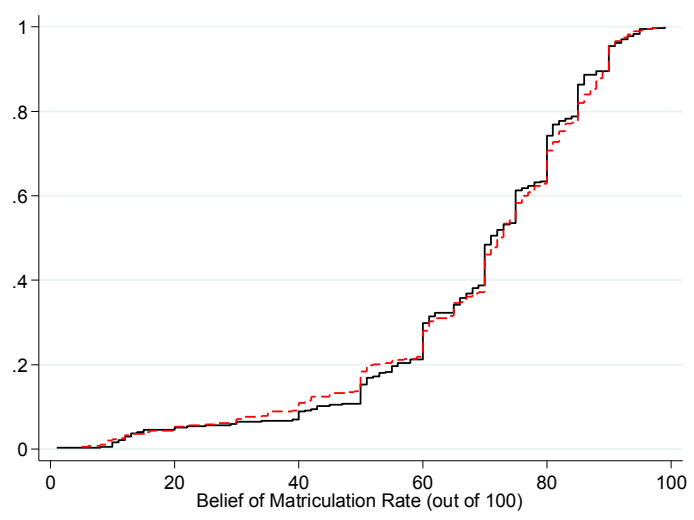

Panel B: Wave 1 Admits

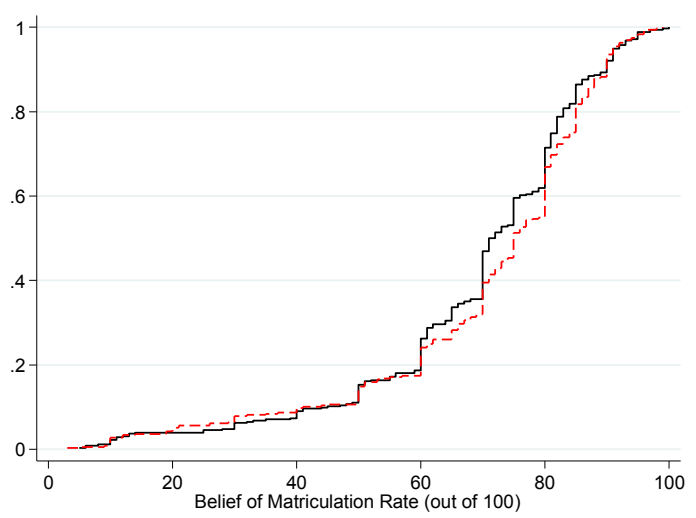

Panel D: Wave 3 Admits
Social Information is dashed $(---)$, Control is solid (—).

Panels show the percentage of subjects who report a matriculation belief weakly smaller than the belief on the $\mathrm{x}$-axis. Subjects in Wave-Specific treatment excluded.

Wave 1 admits were admitted 31 weeks before the survey. Wave 2 admits were admitted 21 weeks before the survey. Wave 3 admits were admitted 14 weeks before the survey. Wave 4 admits were admitted 9 weeks before the survey.

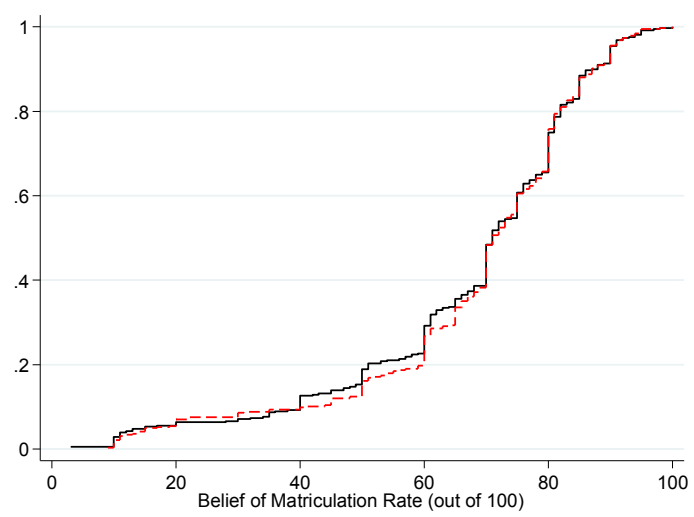

Panel C: Wave 2 Admits

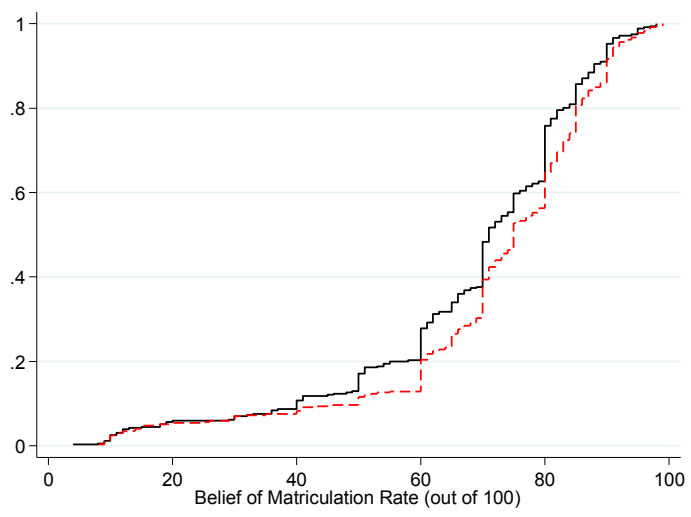

Panel E: Wave 4 Admits

Figure A2: Cumulative Beliefs of Matriculation Rates by Wave and Treatment 
Table A3: Predicting Initial Commitment to TFA with Survey Responses

\begin{tabular}{|c|c|c|c|c|}
\hline & \multicolumn{4}{|c|}{$\begin{array}{c}\text { Linear Probability Model } \\
(\text { Initial Commitment to TFA = 1) }\end{array}$} \\
\hline & (1) & (2) & (3) & (4) \\
\hline \multirow[t]{2}{*}{ Employment Prospects } & 0.08 & & & 0.05 \\
\hline & $(0.01)$ & & & $(0.01)$ \\
\hline \multirow{2}{*}{$\begin{array}{l}\text { Graduate school } \\
\text { Prospects }\end{array}$} & & 0.07 & & 0.03 \\
\hline & & $(0.01)$ & & $(0.01)$ \\
\hline \multirow[t]{2}{*}{ TFA Impact on Students } & & & 0.07 & 0.05 \\
\hline & & & $(0.01)$ & $(0.01)$ \\
\hline Observations & 2,968 & 2,967 & 2,960 & 2,959 \\
\hline
\end{tabular}

Table shows Linear Probability Model (OLS) regression results showing how responses to our survey questions about the value of TFA (each measured on a 7-point Likert scale) predict the decision to accept the offer to join TFA. Subjects in the WaveSpecific Information treatment are excluded. Controls included: wave dummies. Robust standard errors are in parentheses. 
Table A4: Effect of Treatment on Other Survey Questions

\begin{tabular}{lccc|ccc}
\hline & \multicolumn{5}{c}{ Dependent Variable: Likert scale points (1 to 7) } \\
\cline { 2 - 6 } & $\begin{array}{l}\text { Employ. } \\
\text { Prospects }\end{array}$ & $\begin{array}{c}\text { Graduate } \\
\text { School } \\
\text { Prospects }\end{array}$ & $\begin{array}{c}\text { TFA } \\
\text { Impact }\end{array}$ & $\begin{array}{c}\text { Employ. } \\
\text { Prospects }\end{array}$ & $\begin{array}{c}\text { Graduate } \\
\text { School } \\
\text { Prospects }\end{array}$ & TFA Impact \\
\cline { 2 - 6 } & All waves & \multicolumn{3}{c}{ Waves 3 and 4 Only } \\
& $(1)$ & $(2)$ & $(3)$ & $(4)$ & $(5)$ & $(6)$ \\
\hline Social & 0.02 & 0.03 & 0.03 & 0.03 & 0.07 & 0.04 \\
Information & $(0.03)$ & $(0.03)$ & $(0.03)$ & $(0.05)$ & $(0.05)$ & $(0.05)$ \\
\hline Observations & 2,968 & 2,967 & 2,960 & 1,438 & 1,438 & 1,431 \\
\hline
\end{tabular}

Table shows Linear Probability Model (OLS) regression results showing how responses to our survey questions about the value of TFA (each measured on a 7-point Likert scale) predict the decision to accept the offer to join TFA. Subjects in the WaveSpecific Information treatment are excluded. Controls included: wave dummies. Robust standard errors are in parentheses. 